\title{
Kinking of the aorta with calcific aortic valve stenosis
}

\author{
TER U I I UKA W A \\ From the Regional Cardiac Unit, Papworth Hospital, and Addenbrooke's Hospital, Cambridge
}

Kinking or buckling of the aorta is uncommon. The combination of a kinked aorta with severe calcific aortic valve stenosis is very unusual. The importance of recognizing kinking of the aorta lies in the fact that it can mimic other more serious conditions, such as a mediastinal tumour, aortic aneurysm, or coarctation. One of the earliest cases reported was diagnosed as Hodgkin's disease of the superior mediastinal glands ; radiotherapy did not reduce the mass. Thoracotomy revealed the sharply kinked aorta, and the apex of the kink was sited at the insertion of a short patent ductus arteriosus. The aorta was not narrowed (Souders, Pearson, and Adams, 1951).

Systolic murmurs which occur in this condition are usually not indicative of a haemodynamic abnormality. In one series of 10 patients, nine had basal systolic murmurs. The murmurs in these patients were attributed to turbulence of blood in the region of the aortic kink (Bruwer and Burchell, 1956). Co-existent congenital heart disease must not be overlooked in these patients. Associated patent ductus arteriosus (Souders et al., 1951), ventricular septal defect (Di Guglielmo and Guttadauro, 1955), dilated aortic sinus aneurysm (Steinberg, 1956), and, more recently, congenital aortic valve stenosis in four cases, one with additional fibroelastosis (Steinberg and Hagstrom, 1962), have been reported. Other patients with systolic murmurs may have had bicuspid aortic valves (Bentivoglio, Sagarminaga, Uricchio, and Goldberg, 1960).

\section{CASE REPORT}

V. R., a 53-year-old stone-mason, was admitted to hospital in November 1964 complaining of dyspnoea on exertion for one year and angina for three months. There was no histury of rheumatic fever or chorea and there was no family history of heart disease. For the past 20 years he had had infrequent attacks of gout.

On examination the patient appeared older than his years. The brachial pulses were small, slow rising, and anacrotic in type. The pulse rate was 76 per minute and the rhythm was regular. Blood pressure in both arms was $120 / 90 \mathrm{~mm}$. Hg. There was no delay in femoral artery pulsation. The carotids were not visible. The jugular venous pressure was normal. The left ventricle was palpable and dynamic. A moderately loud aortic systolic ejection murmur was heard, but there was no thrill. The second sound was single. There was a faint early diastolic murmur. The lungs were clear. The patient had bilateral nerve deafness. Rectal examination revealed a normal prostate.

A full blood count was normal. Blood urea ranged from 46 to $63 \mathrm{mg} . / 100 \mathrm{ml}$. Urea clearance was $81 \%$ of average normal. Urine culture was negative. The urine was concentrated to a specific gravity of $1 \cdot 020$. Uric acid was $4.5 \mathrm{mg}$. $/ 100 \mathrm{ml}$. An intravenous pyelogram showed kidneys equal in size but rather small, and showed poor concentration of the contrast media.

An electrocardiogram showed sinus rhythm, left axis deviation, and left ventricular hypertrophy and strain (Fig. 1). A chest radiograph in the posteroanterior view revealed a prominent projection of the aortic knuckle to the left (Fig. 2). A tomogram of the aortic arch in this projection revealed that the projecting mass was continuous with the aorta (Fig. 3). The lateral projection showed kinking of the aortic arch. This was confirmed in the left anterior oblique view (Fig. 4). The left ventricle is moderately enlarged and the ascending aorta is dilated. Moderate aortic valve calcification is seen in the lateral view.

Simultaneous left brachial artery and left ventricular punctures showed a systolic gradient of $109 \mathrm{~mm}$. $\mathbf{H g}$ (Fig. 5). The brachial artery pressure was 120/60 and the left ventricular pressure $229 /-11$ to $26 \mathrm{~mm}$. $\mathbf{H g}$. Withdrawal trace across the aortic valve showed a single pressure change at valve level.

\section{DISCUSSION}

Steinberg and Hagstrom (1962) first reported the combination of congenital aortic stenosis with kinking of the aorta in four young patients. One patient showed a stenotic bicuspid aortic valve. No haemodynamic findings were given. The patient presented here showed proved severe calcific aortic valve stenosis, probably congenital in origin since there was no rheumatic history, in association with kinking of the aorta.

The diagnosis of kinking of the aorta can be made radiologically with straight films taken in 
FIG. 1. Electrocardiogram showing left ventricular hypertrophy and strain.
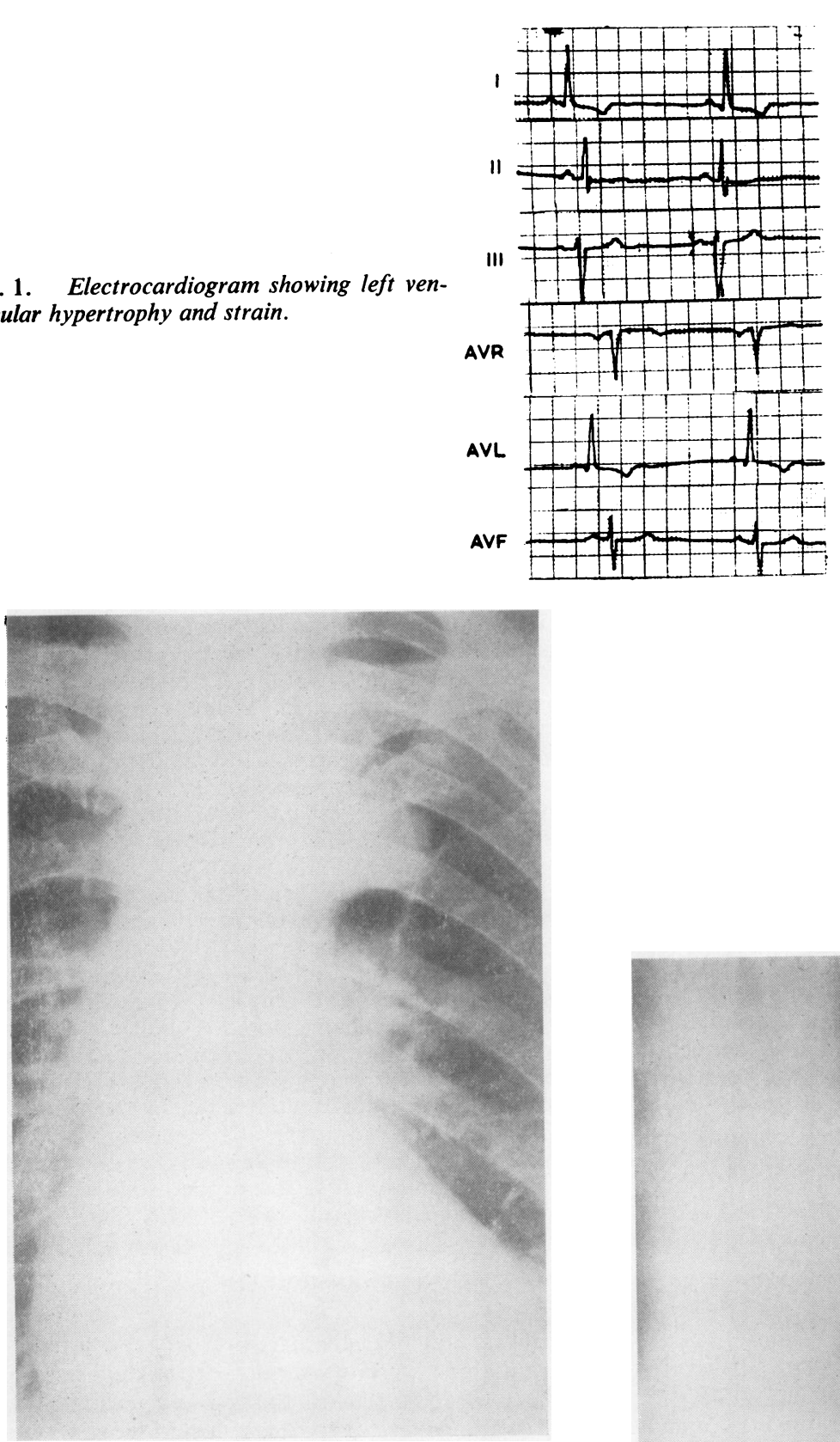

FIG. 2 .

FIG. 2. Postero-anterior view of the heart revealing prominent projection of the aortic knuckle to the left.

FIG. 3. A tomogram of the aortic arch in the posteroanterior projection showed that the projecting mass was continuous with the aorta.
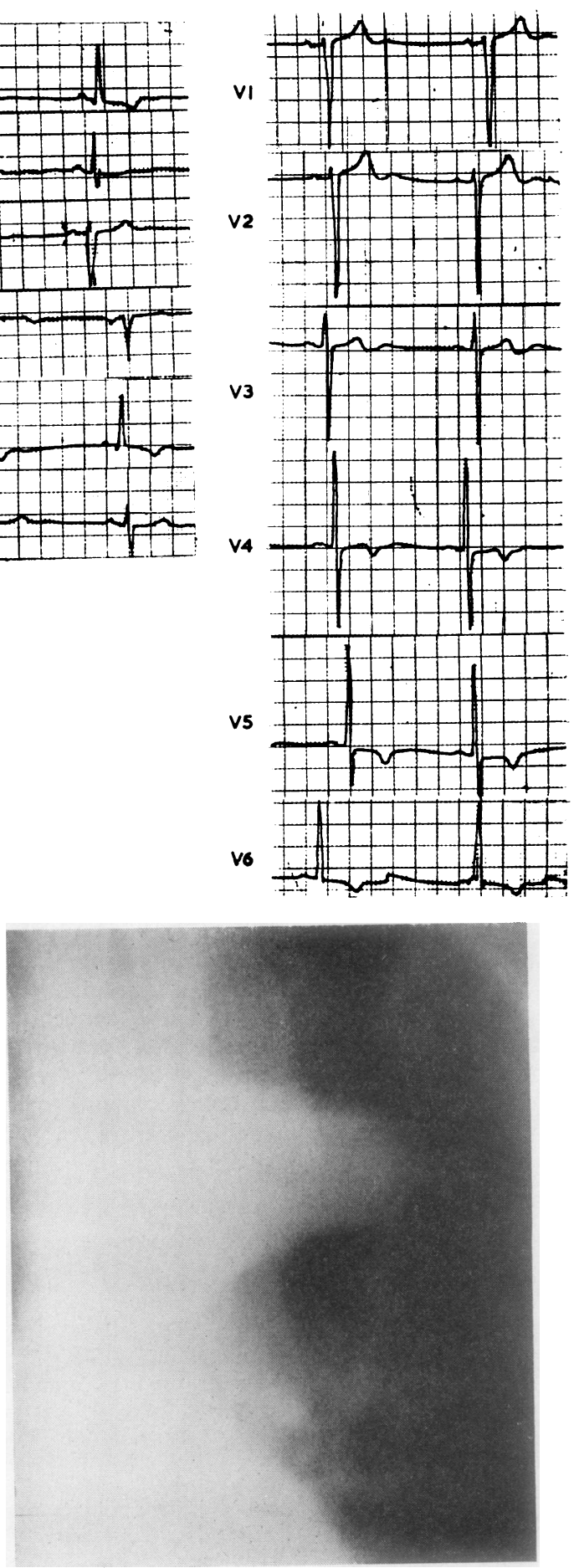

FIG. 3 . 


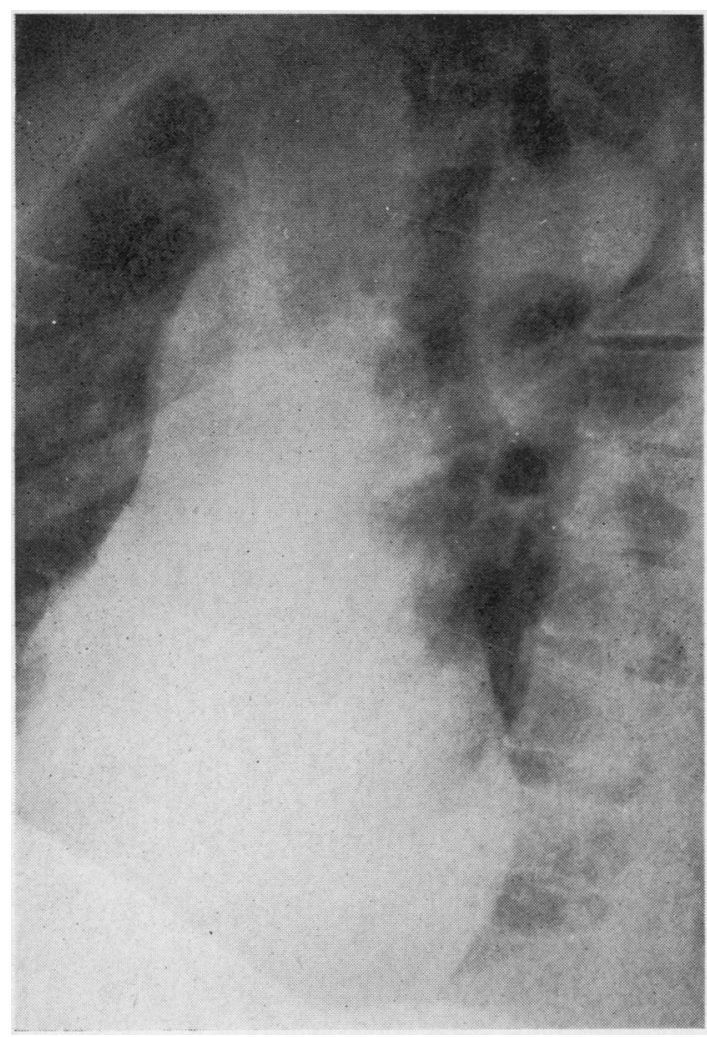

FIG. 4.

the left anterior oblique and right anterior oblique positions as well as in the postero-anterior position. Barium swallow in the postero-anterior and left anterior oblique positions will help. Tomography in the left anterior oblique position may also help (Pattinson and Grainger, 1959).

Opinion is divided between the congenital theory and the developmental theory of the pathogenesis of the condition. In the usual case of unfolding of the aorta, the arch takes a wide smooth sweep over the left main bronchus and down the left paravertebral area. As the arc increases, the ligamentum arteriosum stretches or degenerates without limiting the stretch. If the ligamentum did not stretch, an elongated or tortuous aorta would be buckled at the site of insertion of the ligamentum. This tendency increases with hypertension and with arteriosclerosis. It would be difficult to explain the kinking in our patient in the presence of severe aortic stenosis on this developmental basis.

According to the congenital theory, abnormalities occur during formation of the aortic arch
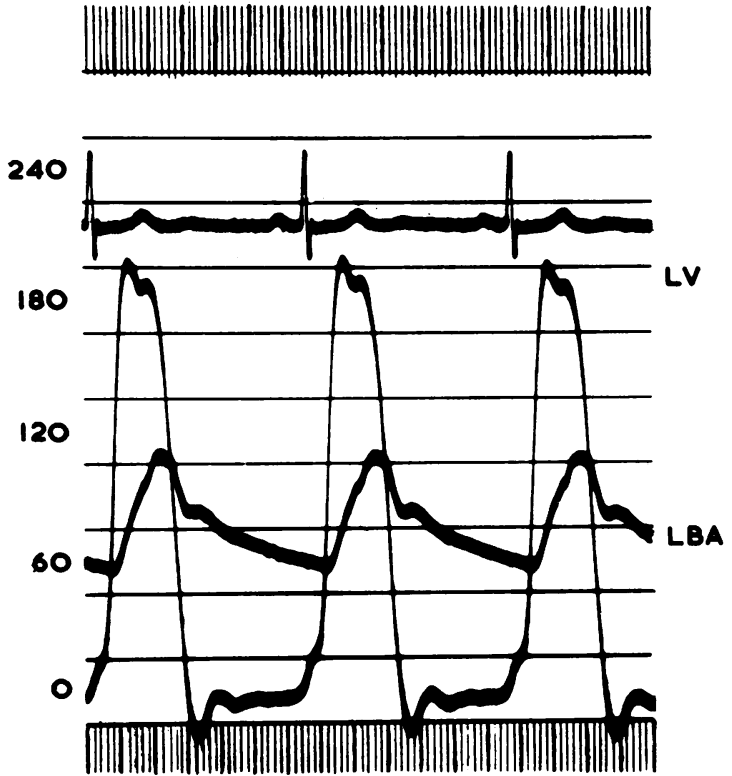

FIG. 5 .

FIG. 4. Left anterior oblique view with kinked aorta showing posteriorly.

FIG. 5. Left ventricular pressure was 229/-11 to 26 and left brachial artery pressure $120 / 60$, giving a peak systolic gradient of $109 \mathrm{~mm}$. $\mathrm{Hg}$.

from the aortic roots. The aortic arch may become abnormally long and twist at the point of insertion of the ligamentum arteriosum (Steinberg and Hagstrom, 1962). In addition, the kinking of the congenitally buckled aortic arch will be increased in later life by arteriosclerosis and hypertension and will become noticeable in middle and older age (Pattinson and Grainger, 1959).

\section{SUMMARY}

The case of a 53-year-old man with severe calcific aortic stenosis and kinking of the arch of the aorta is described. In the absence of a history of rheumatic fever, and with involvement of the aortic valve only, it is considered likely that the aortic valve stenosis was congenital in origin. If this were so, the kinking of the aorta was almost certainly congenital in origin, though possibly aggravated by atherosclerosis.

I am grateful to Dr. H. A. Fleming, under whose care this patient was investigated, for suggesting this 
report, and to Dr. D. McG. Gregg for help with the selection of the chest radiographs.

\section{REFERENCES}

Bentivoglio, L. G., Sagarminaga, J., Uricchio, J., and Goldberg, H. (1960). Congenital bicuspid aortic valves: a clinical and haemo dynamic study. Brit. Heart J., 22, 321.

Bruwer, A. J., and Burchell, H. B. (1956). Kinking of aortic arch (pseudocoarctation, subclinical coarctation). J. Amer. med. Ass.. 162, 1445.
Di Guglielmo, L., and Guttadauro, M. (1955). Kinking of the aorta: report of two cases. Acta radiol. (Stockh.), 44, 121.

Pattinson, J. N., and Grainger, R. G. (1959), Congenital kinking of the aortic arch. Brit. Heart J., $21,555$.

Souders, C. R., Pearson, C. M., and Adams, H. D. (1951). An aortic deformity simulating mediastinal tumor: a subclinical form of coarctation. Dis. Chest, 20, 35.

Steinberg, I. (1956). Aneurysm of the aortic sinuses with pseudocoarctation of the aorta. Brit. Heart J., 18, 85.

and Hagstrom, J. W. C. (1962). Congenital aortic valvular stenosis and pseudocoarctation ('kinking, buckling') of the arch of the aorta; report of four cases including an autopsy study on one case with parietal endocardial fibrosis and fibroelasiosis. one case with pariet
Circulation, $25,545$. 